\title{
Public-private partnerships need honest brokering
}

\author{
Michel Goldman
}

\begin{abstract}
Given the current challenges in research and development, it's increasingly apparent that collaboration between large pharmaceutical companies, academic teams and biotechnology enterprises is essential for converting basic biomedical discoveries into lifesaving medicines. But these partnerships work best when a neutral third party helps foster them.
\end{abstract}

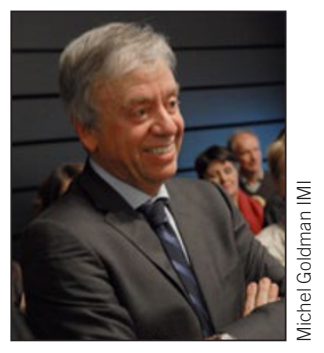

A trickling pipeline of new products at many pharmaceutical companies has led to a paradigm shift in the industry's research and development (R\&D) strategy. Indeed, the integrated R\&D model in which every step of drug development is conducted in-house has proved largely inefficient in delivering the novel therapies needed to address major health challenges. Therefore, this model is being progressively replaced by open innovation networks that allow the leveraging of external pools of knowledge, especially in universities and biotechnology companies ${ }^{1}$.

The pharmaceutical industry realizes that the best approach is to apply an open innovation concept to precompetitive research that encourages companies to share expertise. These principles were the cornerstones of the Critical Path Initiative launched by the US Food and Drug Administration in 2004, which led to the creation of the Critical Path Institute, an Arizona-based nonprofit dedicated to fostering collaborations between industry, academia and regulators ${ }^{2}$.

Across the pond, the Innovative Medicines Initiative (IMI), a public-private partnership between the EU and the European Federation of Pharmaceutical Industries and Associations, is a prototypic example of an organization created to support open innovation and pre-competitive research in the pharmaceutical sector. It has raised awareness about the principles of open collaboration and has launched several education and training programs for scientists from industry or academia interested in drug development and regulatory matters. Indeed, the next IMI call for proposals will aim at setting up a European center for collecting and screening promising pharmaceutical compounds from both public and private sources.

Operating since 2009 with a budget of $€ 2$ billion ( $\$ 2.7$ billion) to be spent until end of 2017, IMI has launched 30 consortia to develop standardized models of disease and to share large clinical data sets, among many other aims. The groups have already reported considerable achievements in areas such as schizophrenia, diabetes, asthma and drug safety. Additional large-scale interdisciplinary projects will be launched this year to address public health issues such as autism, vaccine safety and antimicrobial resistance. These new activities will be conducted by a critical mass of public and private partners after in-depth consultation of regulatory authorities.

The consortia, and indeed all of IMI's activities, are guided by experts coming from the public and private realms. For public-private consortia to work efficiently and successfully, however, they must be managed by a neutral and autonomous organization acting as an honest broker. Such a neutral body ensures that the partners who benefit from public funding-for example, universities and smalland medium-sized enterprises-are selected through a fair and transparent competition, rather than through preexisting connections. For this reason, IMI organizes a competitive process to identify the best partners to match with the pharmaceutical companies that, for their part, invest considerable resources in the projects, propose the research topics and most often coordinate the projects.

This leading role of industry, which distinguishes IMI from most other public-private partnerships, guarantees the optimal exploitation of the knowledge created and its dissemination by the research consortia. As an example, within one of the IMI consortia for diabetes, the optimal exploitation of the first human beta cell line useable for the development of antidiabetic drugs ${ }^{4}$ was made possible by the partnership between the academic team that made the basic discovery, a small enterprise that commercializes the cell product and the large pharmaceutical enterprises that will develop drug screening assays relying on this innovative tool.

Ensuring that consortia operate in a balanced manner in terms of intellectual property and allocation of resources requires a neutral party that can act as a referee whenever needed. To address this need, IMI facilitates consortium agreements by playing the role of impartial broker. A key mission of a neutral body such as IMI is, of course, to ensure the sound management and allocation of public funds in the interest of both industry and society. Here, IMI develops performance indicators suited to measure the added value of public-private partnerships ${ }^{5}$. As an example, IMI is closely monitoring license agreements and the creation of spin-off companies derived from its projects.

One of the most important missions of the managers of publicprivate partnerships is to maintain the interest and commitments of the funding sources-and to guarantee the transparency of the endeavor. This is especially relevant to the information conveyed to patients about the different aspects of drug development, a topic of a recent IMI project. Overall, a neutral organizer is key to ensure the sustainability of public-private partnerships and to restore trust in and among the stakeholders committed to the development of innovative therapies.

Michel Goldman is the executive director of the Innovative Medicine Initiative in Brussels, Belgium. The opinions expressed in this article do not necessarily reflect the positions and opinions of the European Commission or the European Federation of Pharmaceutical Industries and Associations.

1. Melese, T., Lin, S.M., Chang, J.L. \& Cohen, N.H. Nat. Med. 15, 502-507 (2009).

2. Woodcock, J. \& Woosley, R. Annu. Rev. Med. 59, 1-12 (2008).

3. Strauss, S. Nat. Biotechnol. 28, 631-634 (2010).

4. Ravassard, P. et al. J. Clin. Invest. 121, 3589-3597 (2011)

5. Pardoe, D., Hunter, J. \& Cooke, R. Drug Discov. World 12, 9-17 (2011). 